J. Nonlinear Var. Anal. 4 (2020), No. 3, pp. 439-454

Available online at http://jnva.biemdas.com

https://doi.org/10.23952/jnva.4.2020.3.08

\title{
SHRINKING PROJECTION METHOD FOR SOLVING ZERO POINT AND FIXED POINT PROBLEMS IN BANACH SPACES
}

\author{
LIYA LIU ${ }^{1,2}$ \\ ${ }^{1}$ College of Science, Hangzhou Normal University, Hangzhou 310000, China \\ ${ }^{2}$ School of Mathematical Sciences, University of Electronic Science and Technology of China, Chengdu, China
}

\begin{abstract}
In this article, we propose an algorithm for finding a common element of the set of fixed points of quasi- $\phi_{p}$-nonexpansive mappings and zero points of nonnegative, l.s.c., convex mapping in a real $p$-uniformly convex and uniformly smooth Banach space by means of a modified shrinking projection method. Under some mild assumptions, strong convergence theorems are established. Preliminary numerical examples are performed to support our results.
\end{abstract}

Keywords. Fixed point; Quasi- $\phi_{p}$-nonexpansive; Strong convergence; Shrinking projection; Zero point.

\section{INTRODUCTION}

Let $E$ be a real Banach space with the topological dual space $E^{*}$. Let $J_{p}(p>1)$ denote the generalized duality mapping from $E$ to $2^{E^{*}}$ defined by

$$
J_{p}(x):=\left\{x^{*} \in E^{*}:\left\langle x^{*}, x\right\rangle=\|x\|^{p},\left\|x^{*}\right\|=\|x\|^{p-1}\right\}, \forall x \in E,
$$

where $\langle\cdot, \cdot\rangle$ denotes the duality pairing between $E$ and $E^{*}$. We call that $J_{2}$ is the normalized duality mapping. Because of the Hahn-Banach Theorem, we have that $J_{p}(x)$ is nonempty for any $x \in E$.

Let $f: E \rightarrow \mathbb{R}$ be a convex function. The set $\{x \in X: f(x)<+\infty\}$ is denoted by domf. We assume that the interior of the domain of $f$, intdomf, is nonempty. For any $x \in$ intdomf and $y \in E$, we denote by $f^{\circ}(x, y)$ the right-hand derivative of $f$ at $x$ in the direction $y$, that is,

$$
f^{\circ}(x, y):=\lim _{t \searrow 0} \frac{f(x+t y)-f(x)}{t} .
$$

The function $f$ is called Gâteaux differentiable at $x$ if $\lim _{t \rightarrow 0}(f(x+t y)-f(x)) / t$ exists for any $y$. In this case, $f^{\circ}(x, y)$ coincides with $\nabla f(x)$, the value of the gradient $\nabla f$ of $f$ at $x$, see $[17,18]$. Let $f: E \rightarrow \mathbb{R}$ be a convex function which is Gâteaux differentiable for any $x \in$ intdomf. The function $D_{f}: \operatorname{dom} f \times \operatorname{int} \operatorname{dom} f \rightarrow[0,+\infty)$, defined by

$$
D_{f}(y, x)=f(y)-f(x)-\langle\nabla f(x), y-x\rangle,
$$

E-mail address: liya42@qq.com.

Received August 10, 2020; Accepted October 30, 2020.

(C)2020 Journal of Nonlinear and Variational Analysis 
is called the Bregman distance with respect to $f$, see [3]. Let us recall a function $f_{p}: E \rightarrow \mathbb{R}$ by $f_{p}(x)=\frac{1}{p}\|x\|^{p}$, where $1<p<\infty$. In this case, the Bregman distance associated with $f_{p}(\cdot)$ reduces to

$$
\phi_{p}(y, x)=\frac{1}{q}\|y\|^{p}-\left\langle J_{p} y, x\right\rangle+\frac{1}{p}\|x\|^{p},
$$

where $x, y \in E, 1<p<\infty$ satisfies $\frac{1}{p}+\frac{1}{q}=1$ with $q>1$. Under the assumption that $E$ is a Hilbert space, we have that $f_{2}(x)=\frac{1}{2}\|x\|, \forall x \in E$ and $J_{2}$ is an identity mapping, then the Bregman distance associated with $f_{2}(\cdot)$ reduces to $\phi_{2}(x, y)=\frac{1}{2}\|x-y\|^{2}, \forall x, y \in E$.

We define a function $V_{p}: E^{*} \times E \rightarrow[0,+\infty)$ associated with $f_{p}(x)=\frac{1}{p}\|x\|^{p}(1<p<\infty)$ by $V_{p}(y, x)=\frac{1}{q}\|y\|^{q}-\langle y, x\rangle+\frac{1}{p}\|x\|^{p}, \forall x \in E, y \in E^{*}$, see [1]. Recall that $V_{p}$ is nonnegative and

$$
V_{p}(y, x)=\phi_{p}\left(J_{p}^{-1}(y), x\right), \forall x \in E, y \in E^{*} .
$$

By making use of the subdifferential inequality, we obtain that

$$
V_{p}(y, x)+\left\langle z, J_{p}^{-1}(y)-x\right\rangle \leq V_{p}(y+z, x), \forall x \in E, y, z \in E^{*},
$$

see $[9,12]$. Since $V_{p}$ is convex in the first variable, hence we have that for all $z \in E$,

$$
\sum_{i=1}^{N} t_{i} \phi_{p}\left(x_{i}, z\right) \geq \phi_{p}\left(J_{p}^{-1}\left(\sum_{i=1}^{N} t_{i} J_{p}\left(x_{i}\right)\right), z\right),
$$

where $\left(x_{i}\right)_{i=1}^{N} \subset E$ and $\left(t_{i}\right)_{i=1}^{N} \subset(0,1)$ with $\sum_{i=1}^{N} t_{i}=1$; see [10].

The modulus of convexity of $E$ is the function $\tau:(0,2] \rightarrow[0,1]$ defined as follows

$$
\tau(\omega)=\inf \left\{1-\left\|\frac{x+y}{2}\right\|:\|x\|=\|y\|=1 ;\|x-y\|=\omega\right\} .
$$

We call that $E$ is uniformly convex if and only if $\tau(\omega)>0, \forall \omega \in(0,2]$. Let $\lambda>0$. We say that $E$ is $\lambda$-uniformly convex if, there exists a positive constant $\delta$ such that $\tau(\omega) \geq \delta \omega^{\lambda}, \forall \omega \in(0,2]$. Let us define a unit sphere of $E$ by $\mathscr{B}(E):=\{x \in E:\|x\|=1\}$. We say that $E$ is strictly convex if, $\frac{\|x+y\|}{2}<1, \forall x, y \in \mathscr{B}(E), x \neq y$. We say that if $E$ is a strictly convex and reflexive Banach space with a strictly convex dual, one sees that the duality mapping from $E^{*}$ into $E$ satisfies that $\left(J_{p}\right)^{-1} J_{p}=I_{E}$ and $J_{p}\left(J_{p}\right)^{-1}=I_{E^{*}}$, see [8]. On the other hand, we say that the norm of $E$ is Gâteaux differentiable iff the $\operatorname{limit} \lim _{s \rightarrow 0} \frac{\|x+s y\|-\|x\|}{s}$ exists for any $x, y \in \mathscr{B}(E)$. In this case, $E$ is said to be a smooth Banach space. Recall that if $E$ is smooth, then $J_{p}$ is single valued and $J_{p}(x):=\|x\|^{p-2} J(x), \forall x \neq 0$, see [29]. The modulus of smoothness of $E$ is a function $\rho_{E}:[0, \infty) \rightarrow[0, \infty)$ defined by

$$
\rho_{E}(\boldsymbol{l}):=\sup \left\{\frac{\|x+y\|-\|x-y\|}{2}-1:\|x\| \in \mathscr{B}(E),\|y\|=\imath\right\} .
$$

We say that a Banach space $E$ is uniformly smooth iff, $\lim _{\imath \rightarrow 0} \frac{\rho_{E}(l)}{\imath}=0$. Given $r>1$. We say that $E$ is $r$-uniformly smooth iff, there exist a positive constant $\kappa$ such that $\kappa \imath^{r} \geq \rho_{E}(l)$. If $E$ is $r$-uniformly smooth, one sees that $r \leq 2$ and $E$ is uniformly smooth [28]. If $E$ is uniformly smooth, we obtain that $J_{p}$ is uniformly continuous on bounded subsets of $E$. If $E$ is uniformly smooth, one obtains that $\phi_{p}$ is norm-to-norm uniformly continuous on bounded sets of $E$.

Let $C$ be a nonempty, closed, and convex subset of a real Banach space $E$. Let us give a mapping $T$ from $C$ into $E$. We denote by $F i x(T)$ the solution set of finding $u \in E$ such that $u=T u$. According to [19], we say that $T: C \rightarrow E$ is 
(i) $\phi_{p}$-nonxpansive if

$$
\phi_{p}(x, y) \geq \phi_{p}(T x, T y), \forall x, y \in C ;
$$

(ii) quasi- $\phi_{p}$-nonexpansive if $\operatorname{Fix}(T) \neq \emptyset$ and

$$
\phi_{p}\left(x^{*}, x\right) \geq \phi_{p}\left(T x, x^{*}\right), \forall x \in C, x^{*} \in F i x(T) .
$$

We assume that $C$ is a nonempty, closed and convex subset of a smooth, strictly convex and reflexive Banach space $E$. We recall that the generalized projection is a mapping $\Pi_{C}: E \rightarrow C$ that assigns to an arbitrary point $x \in E$ the minimum point of $\phi_{p}(x, y)$, that is $\bar{x}=\Pi_{C} x$, where $\bar{x}$ is the solution to the minimization problem

$$
\Pi_{C} x=\arg \min _{y \in C} \phi_{p}(x, y),
$$

see [2]. In the setting of Hilbert spaces, operator $\Pi_{C}$ is the metric (nearest point) projection $P_{C}$. One knows ([29]) $\Pi_{C}$ can be characterized by the following properties, for any $x \in E$,

$$
\left\langle z-\Pi_{C} x, J_{p}(x)-J_{p}\left(\Pi_{C} x\right)\right\rangle \leq 0, \forall z \in C,
$$

from which one sees that

$$
\phi_{p}\left(\Pi_{C} x, z\right) \leq \phi_{p}(x, z)-\phi_{p}\left(x, \Pi_{C} x\right), \forall z \in C .
$$

Fixed point problems find a number of applications in many practical fields, such as, medical imaging, traffic networks etc. Fixed point methods have been applied to monotone variational inequalities, convex optimization problems and convex feasibility problems [5, 6, 23, 24, 25, 26]. There is an extensive literature on numerical approaches to solving the fixed point problems, such as, the Halpern algorithm, the hybrid method and the Mann algorithm. Recently, the iterative constructions of fixed points of nonlinear mappings via the Mann-like method have been extensively investigated by many authors $[7,13,16,21]$. However, it is worthwhile recalling that this method is weakly convergent in the framework of infinite dimensional Hilbert spaces. As we are working in infinite dimensional spaces, strong convergence, which is a convergence in norm topology, is much more essential and desirable than weak convergence, which is a convergence in weak topology.

Now we recall the shrinking projection method proposed by Takahasi, Takeuchi and Kubota in Hilbert spaces [22]. They obtained a strong convergence result of fixed points for nonexpansive mappings ( $\|T x-T y\| \leq\|x-y\|, \forall x, y \in C$ ). To be more precisely, their result reads as follows.

Theorem 1.1. Let $C$ be a nonempty closed convex subset of a Hilbert space H. Suppose that $T: C \rightarrow H$ is a nonexpansive mapping with Fix $(T) \neq \emptyset$. The iterate sequence $\left\{x_{n}\right\}$ is defined by

$$
\left\{\begin{array}{l}
x_{0} \in H \text { chosen arbitrarily, } \\
C_{1}=C, \\
x_{1}=P_{C_{1}} x_{0}, \\
y_{n}=\alpha_{n} x_{n}+\left(1-\alpha_{n}\right) T x_{n}, \\
C_{n+1}=\left\{z \in C_{n}:\left\|y_{n}-z\right\| \leq\left\|x_{n}-z\right\|\right\}, \\
x_{n+1}=P_{C_{n+1}} x_{0}, \forall n \geq 0 .
\end{array}\right.
$$

where $0 \leq \alpha_{n}<a<1, \forall n \in \mathbb{N}$. Then $\left\{x_{n}\right\}$ converges strongly to the fixed point of $T$ nearest to $x_{0}$, as $n \rightarrow \infty$.

As a generalization of the shrinking projection method, Qin, Huang and Wang [14] improved Theorem 3.1 from a Hilbert space to a Banach space. Their result reads as follows. 
Theorem 1.2. Let $E$ be a uniformly smooth and strictly convex Banach space with the KadecKlee property. Let $C$ be a nonempty closed convex subset of $E$. Suppose that $T: C \rightarrow C$ is a closed and quasi- $\phi_{2}$-nonexpansive mapping. Let $\left\{x_{n}\right\}$ be a sequence generated in the following manner

$$
\left\{\begin{array}{l}
x_{0} \in E \text { chosen arbitrarily, } \\
C_{1}=C, \\
x_{1}=\prod_{C_{1}} x_{0}, \\
y_{n}=J_{2}^{-1}\left[\alpha_{n} J_{2} x_{n}+\left(1-\alpha_{n}\right) J_{2} T x_{n}\right], \\
C_{n+1}=\left\{z \in C_{n}: \phi_{2}\left(z, y_{n}\right) \leq \phi_{2}\left(z, x_{n}\right)\right\}, \\
x_{n+1}=\prod_{C_{n+1}} x_{0}, \forall n \geq 0,
\end{array}\right.
$$

where $\left\{\alpha_{n}\right\} \subset[0,1)$ and limsup $\operatorname{su}_{n \rightarrow \infty} \alpha_{n}<1$. Then $\left\{x_{n}\right\}$ converges strongly to the fixed point of $T$ nearest to $x_{0}$, as $n \rightarrow \infty$.

On the other hand, many physically important problems can be modeled by initial value problems expressed as $S x(t)+x^{\prime}(t)=0, x(0)=x_{0}$, where $S$ is a mapping defined in a Banach space. Some examples where such evolution equations occur can be found in the wave, heat and other fields. Under the assumption that $x(t)$ is dependent on $t$, the above problem can be reduced to $S(x)=0$ whose solutions correspond to the equilibrium points of initial value problems. We denote the zero set of $S$ by $S^{-1}(0)$, that is, $S^{-1}(0)=\{z \in d o m S: 0 \in S(z)\}$. Recently, there are a number of numerical methods for solving zero point problems; see, e.g., [4, 7, 15, 20, 21$]$.

Motivated by the works mentioned above and their real applications, we consider the following problem, which consists of

$$
\text { finding } x \in \bigcap_{i=1}^{n} \operatorname{Fix}\left(T_{i}\right) \cap S^{-1}(0) \text {, }
$$

where $T_{1}, T_{2}, \ldots, T_{n}$ are quasi- $\phi_{p}$-nonexpansive mappings, and $S$ is a nonnegative, convex and lower semi-continuous function.

An outline of this paper is organized as follows. In Section 3, we present some lemmas, which are essential to obtain our main convergence theorem. In Section 3, the main convergence theorem is obtained in a real uniformly smooth and $p$-uniformly convex Banach space. In Section 4, numerical examples are presented to support our algorithm.

\section{LEMMAS}

In this section, we list some lemmas, which are essential to obtain our main convergence theorem.

Lemma 2.1. [27] Let $E$ be a r-uniformly smooth Banach space. Then there is a $\chi_{r}>0$ such that $\|x-y\|^{r} \leq\|x\|^{r}-r\left\langle y, J_{r}(x)\right\rangle+\chi_{r}\|y\|^{r}, \forall x, y \in E$.

Lemma 2.2. [20] Let $E$ be a uniformly convex and smooth Banach space, and let $C$ be a closed convex nonempty subset of $E$. Let $T$ be a quasi- $\phi_{p}$-nonexpansive and closed mapping from $C$ into itself. Thus, Fix $(T)$ is a closed and convex subset of $C$.

Lemma 2.3. [27] Let $\varepsilon>0$ and $r>1$ be two real numbers. A Banach space $E$ is uniformly convex if and only if there exists a strictly increasing, continuous and convex function $\theta: \mathbb{R}^{+} \rightarrow \mathbb{R}^{+}$ such that $\theta(0)=0$ and $\forall x, y \in \mathscr{B}_{\varepsilon}:=\{x \in E:\|x\| \leq \varepsilon\}, \alpha \in[0,1],\|\alpha x+(1-\alpha) y\|^{r} \leq \alpha\|x\|^{r}+$ $(1-\alpha)\|y\|^{r}-\Upsilon_{r}(\alpha) \theta(\|x-y\|)$, where $\Upsilon_{r}(\alpha):=\alpha^{r}(1-\alpha)+\alpha(1-\alpha)^{r}$. 
Lemma 2.4. [11] Let $E$ be a real smooth and uniformly convex Banach space. Let $\left\{x_{n}\right\}$ and $\left\{y_{n}\right\}$ be two sequences in $E$. Then $\lim _{n \rightarrow \infty} \phi_{p}\left(x_{n}, y_{n}\right)=0$ if and only if $\lim _{n \rightarrow \infty}\left\|x_{n}-y_{n}\right\|=0$.

\section{MAIN RESUlTS}

Theorem 3.1. Let $E$ be a real uniformly smooth and p-uniformly convex Banach space with dual $E^{*}$, where $1<p<\infty$. Let $q$ be the conjugate of $p$, that is, $p+q=p q$. Let $C$ be a nonempty, closed and convex subset of $E$. Let $T_{i}: C \rightarrow C(i=1,2, \ldots, m)$ be a finite family of closed and quasi- $\phi_{p}$-nonexpansive mappings. Let $S: E \rightarrow \mathbb{R}$ be a nonnegative, convex and lower semi-continuous (l.s.c) function. Let $\left\{x_{n}\right\}$ be a sequence generated by the following algorithm

$$
\left\{\begin{array}{l}
x_{0} \in E \text { chosen arbitrarily, } \\
C_{1}=C \\
x_{1}=\Pi_{C_{1}} x_{0}, \\
z_{n, m}=J_{p}^{-1}\left[a_{n} J_{p} x_{n}+b_{n} J_{p} T_{m} x_{n}+c_{n}\left(J_{p} x_{n}-t_{n} d_{n}\right)\right], \\
C_{n+1}=\left\{u \in C_{n}: \sup _{m \geq 1} \phi_{p}\left(z_{n, m}, u\right) \leq \phi_{p}\left(x_{n}, u\right)\right\}, \\
x_{n+1}=\prod_{C_{n+1}} x_{0},
\end{array}\right.
$$

where $\left\{a_{n}\right\},\left\{b_{n}\right\},\left\{c_{n}\right\} \subseteq(0,1)$ with $a_{n}+b_{n}+c_{n}=1, \forall n \in \mathbb{N}, d_{n}$ is a search direction with respect to $S$ and

$$
t_{n}= \begin{cases}\frac{\rho_{n} S\left(x_{n}\right)^{p-1}}{\left\|d_{n}\right\|^{p}}, & \text { if } d_{n} \neq 0 \\ 0, & \text { otherwise }\end{cases}
$$

for any $\rho_{n} \in\left(0,\left(\frac{q}{\chi_{q}}\right)^{\frac{1}{q-1}}\right)$, with respect of $\chi_{q}$ given in Lemma 2.1. We additionally assume that

(i) $0 \leq c \leq t_{n} \leq \bar{c}$ for some $c, \bar{c} \in \mathbb{R}$ and for any $n \in \Gamma:=\left\{k \in \mathbb{N}: d_{k} \neq 0\right\}$;

(ii) $\inf _{n \in \Gamma}\left[\rho_{n}\left(1-\frac{\rho_{n}^{r-1} \chi_{q}}{q}\right)\right]>0$;

(iii) $S\left(x_{n}\right) \leq\left\langle d_{n}, x_{n}-z\right\rangle, \forall n \in \mathbb{N}$ and $\forall z \in S^{-1}(0)$.

If $\Lambda=\bigcap_{i=1}^{m} F i x\left(T_{i}\right) \cap S^{-1}(0) \neq \emptyset$, then $\left\{x_{n}\right\}$ converges strongly to $z=\Pi_{\Lambda} x_{0}$, as $n \rightarrow \infty$.

Proof. Since $S$ is nonnegative 1.s.c convex function, we find that $S^{-1}(0)$ is convex and closed. From this and Lemma 2.2, we obtain that $\Lambda$ is closed and convex. It ensures that $\Pi_{\Lambda}$ is welldefined. Observe that

$$
\begin{aligned}
C_{n+1} & =\left\{u \in C_{n}: \sup _{m \geq 1} \phi_{p}\left(z_{n, m}, u\right) \leq \phi_{p}\left(x_{n}, u\right)\right\}, \\
& =\bigcap_{m \geq 1}\left\{u \in E: \phi_{p}\left(z_{n, m}, u\right) \leq \phi_{p}\left(x_{n}, u\right)\right\} \cap C_{n}, \\
& =\bigcap_{m \geq 1}\left\{u \in E:\left\langle J_{p} x_{n}-J_{p} z_{n, m}, u\right\rangle \leq \frac{1}{r}\left\|x_{n}\right\|^{p}-\frac{1}{r}\left\|z_{n, m}\right\|^{p}\right\} \cap C_{n} .
\end{aligned}
$$

This implies that $C_{n+1}$ is closed and convex. So, $\Pi_{C_{n+1}}$ is well-defined, for $n \in \mathbb{N}$. Let us now fix $\hat{x} \in \Omega$ and show $\Lambda \subset C_{n}$. Recalling the expression of $z_{n, m}$, it follows from (1.1) that

$$
\begin{aligned}
& \phi_{p}\left(z_{n, m}, \hat{x}\right) \\
& =\phi_{p}\left(J_{p}^{-1}\left[a_{n} J_{p} x_{n}+b_{n} J_{p} T_{m} x_{n}+c_{n}\left(J_{p} x_{n}-t_{n} d_{n}\right)\right], \hat{x}\right) \\
& =\phi_{p}\left(J_{p}^{-1}\left[\left(1-c_{n}\right)\left(\frac{a_{n}}{1-c_{n}} J_{p} x_{n}+\frac{b_{n}}{1-c_{n}} J_{p} T_{m} x_{n}\right)+c_{n}\left(J_{p} x_{n}-t_{n} d_{n}\right)\right], \hat{x}\right) .
\end{aligned}
$$


Setting briefly $y_{n, m}=J_{p}^{-1}\left(\frac{a_{n}}{1-c_{n}} J_{p} x_{n}+\frac{b_{n}}{1-c_{n}} J_{p} T_{m} x_{n}\right)$, we immediately find that

$$
\phi_{p}\left(z_{n, m}, \hat{x}\right) \leq\left(1-c_{n}\right) \phi_{p}\left(y_{n, m}, \hat{x}\right)+c_{n} \phi_{p}\left(J_{p}^{-1}\left(J_{p} x_{n}-t_{n} d_{n}\right), \hat{x}\right)
$$

By use of Lemma 2.1 and the definition of $t_{n}$, one has that

$$
\begin{aligned}
& \phi_{p}\left(J_{p}^{-1}\left(J_{p} x_{n}-t_{n} d_{n}\right), \hat{x}\right) \\
= & \frac{1}{q}\left\|J_{p} x_{n}-t_{n} d_{n}\right\|^{q}-\left\langle J_{p} x_{n}, \hat{x}\right\rangle+t_{n}\left\langle d_{n}, \hat{x}\right\rangle+\frac{1}{p}\|\hat{x}\|^{p} \\
\leq & \frac{1}{q}\left(\left\|J_{p} x_{n}\right\|^{q}-q t_{n}\left\langle d_{n}, x_{n}\right\rangle+t_{n}^{q} \chi_{q}\left\|d_{n}\right\|^{q}\right)-\left\langle J_{p} x_{n}, \hat{x}\right\rangle+t_{n}\left\langle d_{n}, \hat{x}\right\rangle+\frac{1}{p}\|\hat{x}\|^{p} \\
= & \frac{1}{r}\left\|x_{n}\right\|^{p}-\left\langle J_{p} x_{n}, \hat{x}\right\rangle+\frac{1}{p}\|\hat{x}\|^{p}-t_{n}\left\langle d_{n}, x_{n}-\hat{x}\right\rangle+\frac{t_{n}^{q} \chi_{q}}{q}\left\|d_{n}\right\|^{q} \\
\leq & \phi_{p}\left(x_{n}, \hat{x}\right)-\rho_{n}\left(1-\frac{\rho_{n}^{q-1} \chi_{q}}{q}\right) \frac{S\left(x_{n}\right)^{p}}{\left\|d_{n}\right\|^{p}} \\
\leq & \phi_{p}\left(x_{n}, \hat{x}\right) .
\end{aligned}
$$

Invoking that $T_{i}(i=1,2, \cdots, m)$ is quasi- $\phi_{p}$-nonexpansive, one finds from Lemma 2.3 that

$$
\begin{aligned}
\phi_{p}\left(y_{n, m}, \hat{x}\right)= & \frac{1}{q}\left\|\frac{a_{n}}{1-c_{n}} J_{p} x_{n}+\frac{b_{n}}{1-c_{n}} J_{p} T_{m} x_{n}\right\|^{q}-\left\langle\frac{a_{n}}{1-c_{n}} J_{p} x_{n}+\frac{b_{n}}{1-c_{n}} J_{p} T_{m} x_{n}, \hat{x}\right\rangle+\frac{1}{p}\|\hat{x}\|^{p} \\
\leq & \frac{1}{q}\left(\frac{a_{n}}{1-c_{n}}\left\|J_{p} x_{n}\right\|^{q}+\frac{b_{n}}{1-c_{n}}\left\|J_{p} T_{m} x_{n}\right\|^{q}-\Upsilon_{q}\left(\frac{a_{n}}{1-c_{n}}\right) \theta\left(\left\|J_{p} x_{n}-J_{p} T_{m} x_{n}\right\|\right)\right) \\
& -\left\langle\frac{a_{n}}{1-c_{n}} J_{p} x_{n}+\frac{b_{n}}{1-c_{n}} J_{p} T_{m} x_{n}, \hat{x}\right\rangle+\frac{1}{p}\|\hat{x}\|^{p} \\
\leq & \frac{a_{n}}{1-c_{n}}\left(\frac{1}{q}\left\|J_{p} x_{n}\right\|^{q}-\left\langle J_{p} x_{n}, \hat{x}\right\rangle+\frac{1}{p}\|\hat{x}\|^{p}\right) \\
& +\frac{b_{n}}{1-c_{n}}\left(\frac{1}{q}\left\|J_{p} T_{m} x_{n}\right\|^{q}-\left\langle J_{p} T_{m} x_{n}, \hat{x}\right\rangle+\frac{1}{p}\|\hat{x}\|^{p}\right) \\
& -\frac{1}{q} \Upsilon_{q}\left(\frac{a_{n}}{1-c_{n}}\right) \theta\left(\left\|J_{p} x_{n}-J_{p} T_{m} x_{n}\right\|\right) \\
\leq & \frac{a_{n}}{1-c_{n}} \phi_{p}\left(J_{p}^{-1}\left(J_{p} x_{n}\right), \hat{x}\right)+\frac{b_{n}}{1-c_{n}} \phi_{p}\left(J_{p}^{-1}\left(J_{p} T_{m} x_{n}\right), \hat{x}\right) \\
& -\frac{1}{q} \Upsilon_{q}\left(\frac{a_{n}}{1-c_{n}}\right) \theta\left(\left\|J_{p} x_{n}-J_{p} T_{m} x_{n}\right\|\right) \\
\leq & \phi_{p}\left(x_{n}, \hat{x}\right)-\frac{1}{q} \Upsilon_{q}\left(\frac{a_{n}}{1-c_{n}}\right) \theta\left(\left\|J_{p} x_{n}-J_{p} T_{m} x_{n}\right\|\right) .
\end{aligned}
$$


By collecting the above results (3.2), (3.3) and (3.4), one finds that

$$
\begin{aligned}
\phi_{p}\left(z_{n, m}, \hat{x}\right) \leq & \left(1-c_{n}\right)\left(\phi_{p}\left(x_{n}, \hat{x}\right)-\frac{1}{q} \Upsilon_{q}\left(\frac{a_{n}}{1-c_{n}}\right) \theta\left(\left\|J_{p} x_{n}-J_{p} T_{m} x_{n}\right\|\right)\right) \\
& +c_{n}\left(\phi_{p}\left(x_{n}, \hat{x}\right)-\rho_{n}\left(1-\frac{\rho_{n}^{q-1} \chi_{q}}{q}\right) \frac{S\left(x_{n}\right)^{p}}{\left\|d_{n}\right\|^{p}}\right) \\
\leq & \phi_{p}\left(x_{n}, \hat{x}\right)-\frac{1-c_{n}}{q} \Upsilon_{r}\left(\frac{a_{n}}{1-c_{n}}\right) \theta\left(\left\|J_{p} x_{n}-J_{p} T_{m} x_{n}\right\|\right) \\
& -c_{n} \rho_{n}\left(1-\frac{\rho_{n}^{q-1} \chi_{q}}{q}\right) \frac{S\left(x_{n}\right)^{p}}{\left\|d_{n}\right\|^{p}} .
\end{aligned}
$$

From this, one concludes that

$$
\phi_{p}\left(z_{n, m}, \hat{x}\right) \leq \phi_{p}\left(x_{n}, \hat{x}\right)
$$

As a consequence, we obtain that $\Lambda \subset C_{n}$. In light of $x_{n}=\Pi_{C_{n}} x_{0}, \forall n \geq 1$, one finds from (1.3) that

$$
\phi_{p}\left(x_{n}, x_{0}\right)=\phi_{p}\left(\Pi_{C_{n}} x_{0}, x_{0}\right) \leq \phi_{p}\left(\hat{x}, x_{0}\right)-\phi_{p}\left(\hat{x}, \Pi_{C_{n+1}} x_{0}\right) \leq \phi_{p}\left(\hat{x}, x_{0}\right), \forall n \geq 1 .
$$

This implies that $\left\{\phi_{p}\left(x_{n}, x_{0}\right)\right\}$ is bounded. According to $x_{n+1}=\Pi_{C_{n+1}} x_{0} \in C_{n+1} \subseteq C_{n}$, we refer that

$$
\phi_{p}\left(x_{n}, x_{0}\right) \leq \phi_{p}\left(x_{n+1}, x_{0}\right), \forall n \geq 1 .
$$

As a result, we immediately have that $\left\{\phi_{p}\left(x_{n}, x_{0}\right)\right\}$ is nondecreasing and bounded. Then the limit $\lim _{n \rightarrow \infty} \phi_{p}\left(x_{n}, x_{0}\right)$ exists. Again, by making use of (1.3), we have that

$$
\begin{aligned}
\phi_{p}\left(x_{n+1}, x_{n}\right) & =\phi_{p}\left(x_{n+1}, \Pi_{C_{n}} x_{0}\right) \leq \phi_{p}\left(x_{n+1}, x_{0}\right)-\phi_{p}\left(\Pi_{C_{n}} x_{0}, x_{0}\right) \\
& =\phi_{p}\left(x_{n+1}, x_{0}\right)-\phi_{p}\left(x_{n}, x_{0}\right)
\end{aligned}
$$

which implies that

$$
\lim _{n \rightarrow \infty} \phi_{p}\left(x_{n+1}, x_{n}\right)=0 .
$$

It follows from (3.7) and Lemma 2.4 that

$$
\lim _{n \rightarrow \infty}\left\|x_{n+1}-x_{n}\right\|=0 .
$$

On the other hand, taking account of (3.6) and (3.8), with the help of Lemma 2.4, we obtain that

$$
\lim _{n \rightarrow \infty}\left\|z_{n, m}-x_{n+1}\right\|=0 \text {. }
$$

Let us give some positive integers $k, l$ with $k \leq l$. By combining $x_{l}=\Pi_{C_{l}} x_{0} \subseteq C_{k}$ with (1.3), we infer that

$$
\begin{aligned}
\phi_{p}\left(x_{k}, x_{l}\right) & =\phi_{p}\left(x_{k}, \Pi_{C_{l}} x_{0}\right) \leq \phi_{p}\left(x_{k}, x_{0}\right)-\phi_{p}\left(\Pi_{C_{l}} x_{0}, x_{0}\right) \\
& =\phi_{p}\left(x_{k}, x_{0}\right)-\phi_{p}\left(x_{l}, x_{0}\right) .
\end{aligned}
$$

Since $\lim _{n \rightarrow \infty} \phi_{p}\left(x_{n}, x_{0}\right)$ exists, we have that (3.10) yields that $\lim _{l \rightarrow \infty} \phi_{p}\left(x_{k}, x_{l}\right)=0$. From Lemma 2.4, we get that $\lim _{l \rightarrow \infty}\left\|x_{k}-x_{l}\right\|=0$. Hence, $\left\{x_{n}\right\}$ is a Cauchy sequence. On the other 
hand, with the help of $\phi_{p}(\cdot, \cdot)$, we derive that

$$
\begin{aligned}
& \phi_{p}\left(x_{n}, \hat{x}\right)-\phi_{p}\left(z_{n, m}, \hat{x}\right) \\
= & \left(\frac{1}{q}\left\|x_{n}\right\|^{p}-\left\langle J_{p} x_{n}, \hat{x}\right\rangle+\frac{1}{p}\|\hat{x}\|^{p}\right)-\left(\frac{1}{q}\left\|z_{n, m}\right\|^{p}-\left\langle J_{p} z_{n, m}, \hat{x}\right\rangle+\frac{1}{p}\|\hat{x}\|^{p}\right) \\
= & \frac{1}{q}\left(\left\|x_{n}\right\|^{p}-\left\|z_{n, m}\right\|^{p}\right)+\left\langle J_{p} z_{n, m}-J_{p} x_{n}, \hat{x}\right\rangle \\
= & \phi_{p}\left(x_{n}, z_{n, m}\right)+\left\langle J_{p} z_{n, m}-J_{p} x_{n}, \hat{x}-z_{n, m}\right\rangle .
\end{aligned}
$$

From the same argument above, we have that

$$
\phi_{p}\left(z_{n, m}, \hat{x}\right)-\phi_{p}\left(x_{n}, \hat{x}\right)=\phi_{p}\left(z_{n, m}, x_{n}\right)+\left\langle J_{p} x_{n}-J_{p} z_{n, m}, \hat{x}-x_{n}\right\rangle .
$$

Combining (3.11) with (3.12), one obtains that

$$
\phi_{p}\left(x_{n}, z_{n, m}\right)+\phi_{p}\left(z_{n, m}, x_{n}\right)=\left\langle J_{p} z_{n, m}-J_{p} x_{n}, z_{n, m}-x_{n}\right\rangle .
$$

As a classical result, one deduces that

$$
\phi_{p}\left(x_{n}, z_{n, m}\right) \leq\left\langle J_{p} z_{n, m}-J_{p} x_{n}, z_{n, m}-x_{n}\right\rangle .
$$

By making use of (3.11) and (3.13), one immediately finds that

$$
\phi_{p}\left(x_{n}, \hat{x}\right)-\phi_{p}\left(z_{n, m}, \hat{x}\right) \leq\left\langle J_{p} z_{n, m}-J_{p} x_{n}, \hat{x}-x_{n}\right\rangle .
$$

Keeping in mind that $E$ is a $p$-uniformly convex and uniformly smooth real Banach space, we have that $J_{p}$ is uniformly norm to norm continuous. Coming back to (3.8) and (3.9), we have that

$$
\lim _{n \rightarrow \infty}\left\|J_{p} z_{n, m}-J_{p} x_{n}\right\|=0 .
$$

Returning to (3.5), together with (3.14), (3.15), we deduce that

$$
\begin{aligned}
& \frac{1-c_{n}}{q} \Upsilon_{r}\left(\frac{a_{n}}{1-c_{n}}\right) \theta\left(\left\|J_{p} x_{n}-J_{p} T_{m} x_{n}\right\|\right)+c_{n} \rho_{n}\left(1-\frac{\rho_{n}^{q-1} \chi_{q}}{q}\right) \frac{S\left(x_{n}\right)^{p}}{\left\|d_{n}\right\|^{p}} \\
\leq & \phi_{p}\left(x_{n}, \hat{x}\right)-\phi_{p}\left(z_{n, m}, \hat{x}\right) \rightarrow 0, \text { as } n \rightarrow \infty .
\end{aligned}
$$

By using successively (3.16) and $\Upsilon_{r}\left(\frac{a_{n}}{1-c_{n}}\right) \neq 0$, we check that

$$
\lim _{n \rightarrow \infty} \theta\left(\left\|J_{p} x_{n}-J_{p} T_{m} x_{n}\right\|\right)=0 \Rightarrow \lim _{n \rightarrow \infty}\left\|J_{p} x_{n}-J_{p} T_{m} x_{n}\right\|=0 .
$$

On the other hand, invoking $\rho_{n} \in\left(0,\left(\frac{q}{\chi_{q}}\right)^{\frac{1}{q-1}}\right)$, we obtain that

$$
\lim _{n \rightarrow \infty} \frac{S\left(x_{n}\right)^{p}}{\left\|d_{n}\right\|^{p}}=0 \Rightarrow \lim _{n \rightarrow \infty} S\left(x_{n}\right)=0 .
$$

Recalling that $J_{p}^{-1}$ is also uniformly norm-to-norm continuous on bounded sets, (3.17) yields that

$$
\lim _{n \rightarrow \infty}\left\|x_{n}-T_{m} x_{n}\right\|=0 .
$$

Observing that $\left\{x_{n}\right\}$ is a Cauchy sequence, we find that there exists $\bar{x} \in E$ such that $\left\{x_{n}\right\}$ converges strongly to $\bar{x}$ as $n \rightarrow \infty$. Owning to (3.19), we have that $\left\{T_{m} x_{n}\right\}$ also converges strongly to $\bar{x}$ as $n \rightarrow \infty$. Recalling that $T_{i}(i=1,2, \cdots, m)$ is closed, we immediately find that $\bar{x} \in \bigcap_{i=1}^{m} F i x\left(T_{i}\right)$. Returning to (3.18), one sees that $\bar{x} \in S^{-1}(0)$. In conclusion, we obtain that $\bar{x} \in \Lambda$. 
Now we are in a position to prove that $\left\{x_{n}\right\}$ converges strongly to an element $z=\Pi_{\Omega} x_{0}$, as $n \rightarrow \infty$. Invoking $x_{n}=\Pi_{C_{n}} x_{0}$, it follows from (1.3) that

$$
\left\langle x_{n}-x, J_{p} x_{0}-J_{p} x_{n}\right\rangle \geq 0, \forall x \in \Lambda .
$$

Taking the limit as $n \rightarrow \infty$ in (3.20), we obtain that

$$
\left\langle\bar{x}-x, J_{p} x_{0}-J_{p} \bar{x}\right\rangle \geq 0, \forall x \in \Lambda .
$$

As a classical result, we conclude that $\bar{x}=\Pi_{\Omega} x_{0}$. Thus, $z=\bar{x}$. It follows that $\left\{x_{n}\right\}$ converges strongly to $z$, as $n \rightarrow \infty$. The proof is completed.

In Theorem 3.1, if direction vector $d_{n}$ coincides with gradient $\nabla S\left(x_{n}\right)$, then we obtain the following corollary.

Corollary 3.1. Let $E$ be a real uniformly smooth and p-uniformly convex Banach space with dual $E^{*}$, where $1<p<\infty$. Let $C$ be a nonempty, closed and convex subset of $E$. Let $T_{i}: C \rightarrow C(i=$ $1,2, \ldots, m)$ be a finite family of closed and quasi- $\phi_{p}$-nonexpansive mappings. Let $S: E \rightarrow \mathbb{R}$ be a nonnegative, convex lower semi-continuous (l.s.c) and Gâteaux differentiable function with the gradient $\nabla S: E \rightarrow E$. Let $\left\{x_{n}\right\}$ be a sequence generated by the following algorithm

$$
\left\{\begin{array}{l}
x_{0} \in E \text { chosen arbitrarily, } \\
C_{1}=C \\
x_{1}=\prod_{C_{1}} x_{0} \\
z_{n, m}=J_{p}^{-1}\left[a_{n} J_{p} x_{n}+b_{n} J_{p} T_{m} x_{n}+c_{n}\left(J_{p} x_{n}-t_{n} \nabla S\left(x_{n}\right)\right)\right] \\
C_{n+1}=\left\{u \in C_{n}: \sup _{m \geq 1} \phi_{p}\left(z_{n, m}, u\right) \leq \phi_{p}\left(x_{n}, u\right)\right\} \\
x_{n+1}=\prod_{C_{n+1}} x_{0}
\end{array}\right.
$$

where $\left\{a_{n}\right\},\left\{b_{n}\right\},\left\{c_{n}\right\} \subseteq(0,1)$ and $a_{n}+b_{n}+c_{n}=1, \forall n \in \mathbb{N}$. If $\Lambda=\bigcap_{i=1}^{m} F\left(T_{i}\right) \cap S^{-1}(0) \neq \emptyset$, then $\left\{x_{n}\right\}$ converges strongly to $z=\Pi_{\Lambda} x_{0}$, as $n \rightarrow \infty$.

\section{NUMERICAL EXPERIMENTS}

In this section, we use two numerical examples to illustrate the computational performance of our proposed algorithm. All the programs are performed in MATLAB2018a on a PC Desktop Intel(R) Core(TM) i5-8250U CPU @ 1.60GHz 1.800 GHz, RAM 8.00 GB.

Example 4.1. In the first experiment, we assume that $E=\mathbb{R}^{N}$ and $C=E$. Let us define $T_{i}: \mathbb{R}^{N} \rightarrow \mathbb{R}^{N}(i=1,2, \ldots, m)$ by $T_{i} x:=\frac{1}{i+1} x(\forall x \in E)$. Meanwhile, we define $S: \mathbb{R}^{N} \rightarrow \mathbb{R}^{N}$ by $S(x):=x^{2}(\forall x \in E)$. Then we check that $T_{i}(i=1,2, \ldots, m)$ is quasi- $\phi_{p}$-nonexpansive. In this case, we observe that $\Lambda=\bigcap_{i=1}^{m} F i x\left(T_{i}\right) \cap S^{-1}(0)=\mathbf{0} \neq \emptyset$.

We use our Algorithm (3.1) to solve Example 4.1 with $N=3, m=3$ and $C_{0}=\mathbb{R}^{N}$. Our parameters are given as follows. We set $a_{n}=b_{n}=c_{n}=\frac{1}{3}$ and $\rho_{n}=0.5(\forall n \in \mathbb{N})$. Our initial value is randomly generated in the range of $(0,1)^{N}$. In this test, we consider four cases (Case I: $N=1$; Case II: $N=10$; Case III: $N=50$; Case IV: $N=100$ ). Let us define a sequence $\left\{E_{n}\right\}$ by $E_{n}=\left\|x_{n}-z\right\|^{2}$ with $z=\mathbf{0}$. In this experiment, the sequence $\left\{E_{n}\right\}$ can be regarded as the iteration error of Algorithm (3.21), due to the fact that the unique solution of $\Lambda$ is $z=\mathbf{0}$. We take $E_{n}<10^{-4}$ as the stopping criteria. Our numerical results are reported in Figure 1 and Figure 2.

In addition, we also tested the numerical behaviors of our algorithm (3.1) with different values of $N$ and $m$. Our numerical results are represented in Figure 3 and Figure 4. 


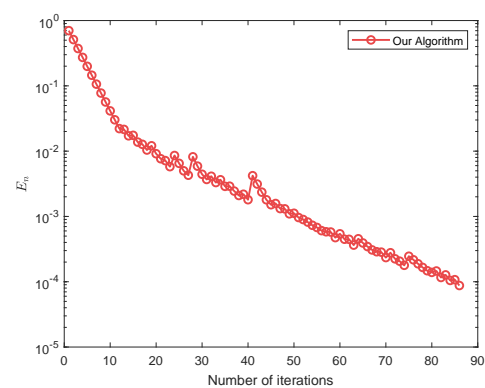

(A) Case I

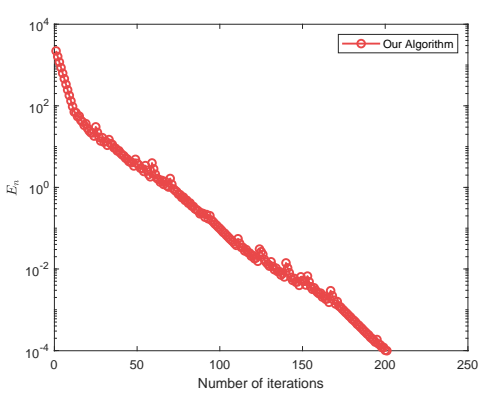

(C) Case III

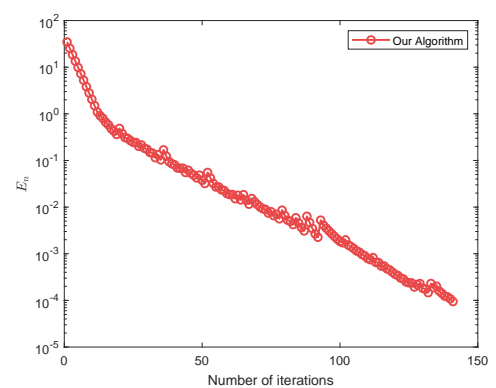

(B) Case II

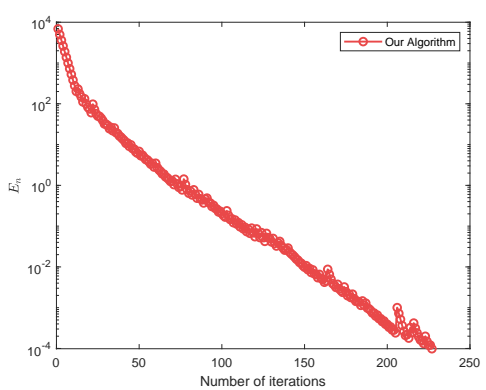

(D) Case IV

FIGURE 1. Numerical behaviors of error sequence $\left\{E_{n}\right\}$ for Example 4.1.

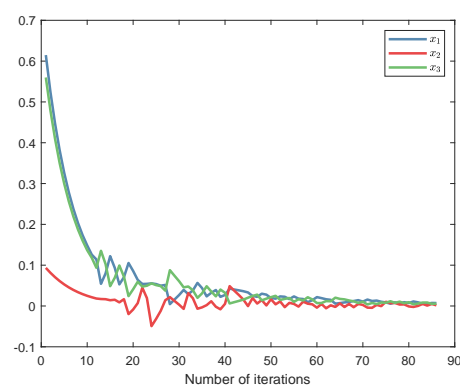

(A) Case I

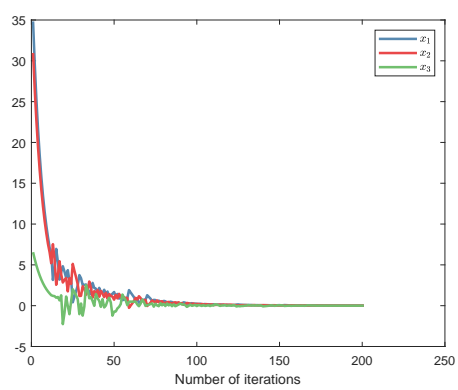

(C) Case III

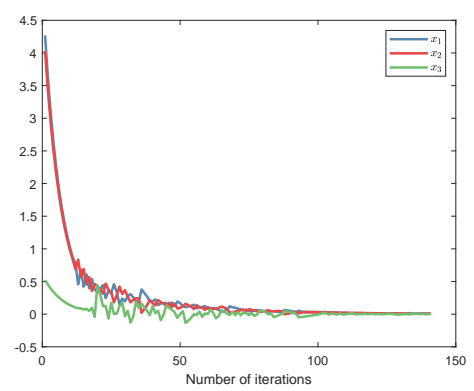

(B) Case II

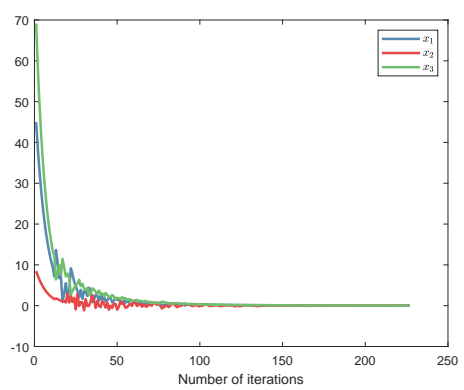

(D) Case IV

FIGURE 2. Numerical behaviors of each component of $x_{n}$ for Example 4.1. 


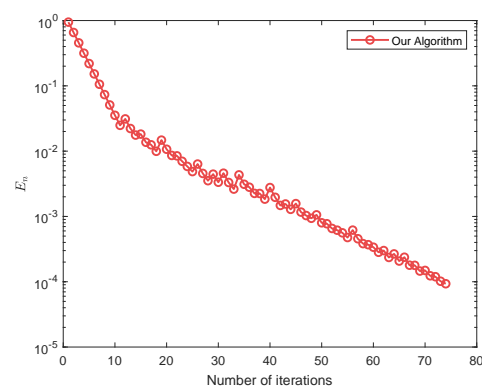

(A) $N=3, m=10$

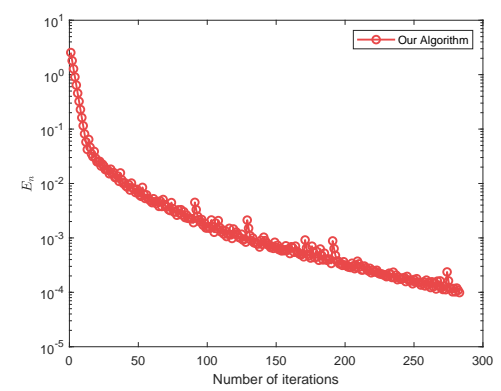

(C) $N=10, m=10$

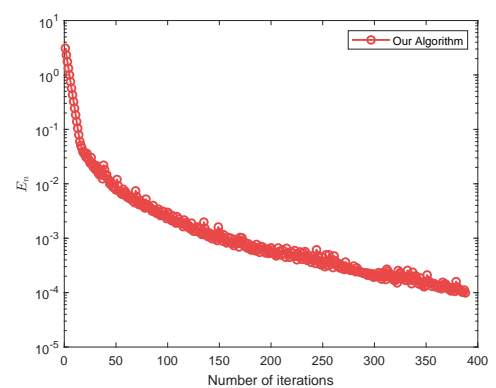

(B) $N=10, m=3$

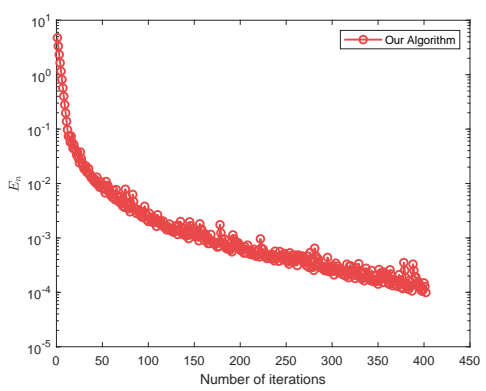

(D) $N=20, m=20$

FIGURE 3. Numerical behaviors of error sequence $\left\{E_{n}\right\}$ for Example 4.1.

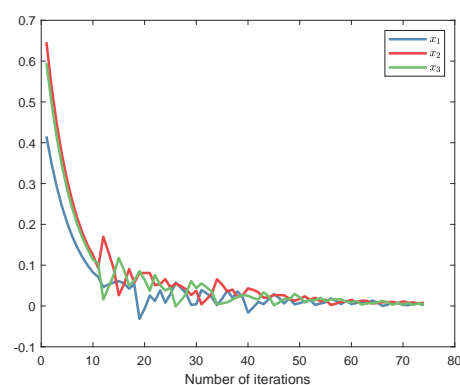

(A) $N=3, m=10$

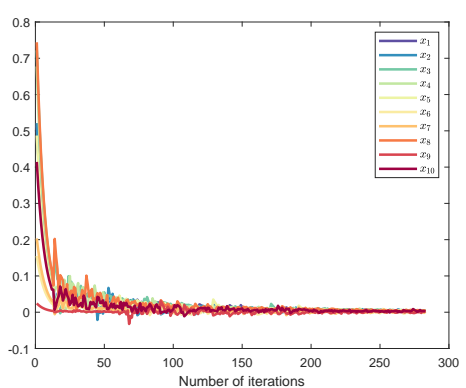

(C) $N=10, m=10$

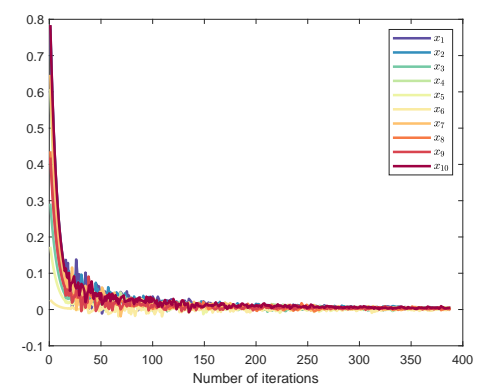

(B) $N=10, m=3$

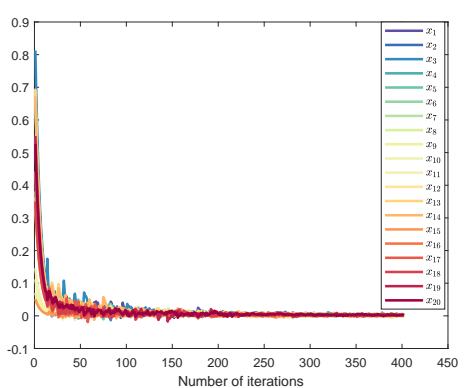

(D) $N=20, m=20$

FIGURE 4. Numerical behaviors of each component of $x_{n}$ for Example 4.1. 


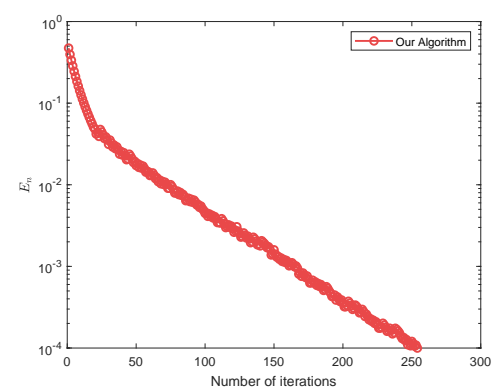

(A) Case I

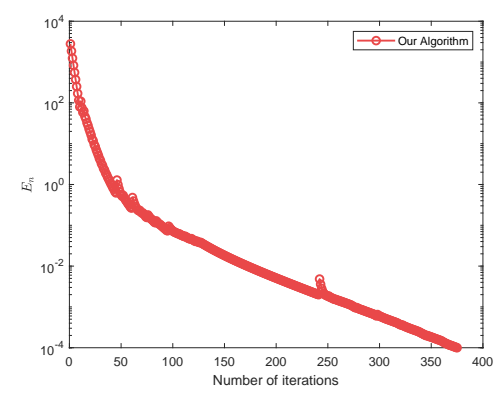

(C) Case III

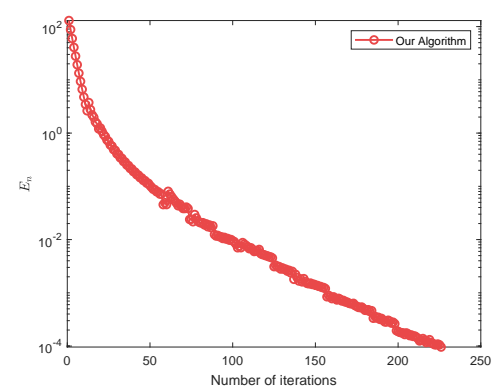

(B) Case II

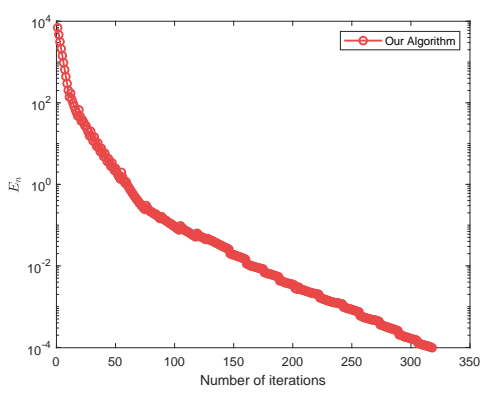

(D) Case IV

FIGURE 5. Numerical behaviors of error sequence $\left\{E_{n}\right\}$ for Example 4.2.

Example 4.2. Let us consider a convex feasibility problem with $E=\mathbb{R}^{N}$ and $C=E$. Given any nonempty closed convex set $Q_{i} \subset \mathbb{R}^{N}(i=1, \ldots, m)$ with $Q:=\bigcap_{i=1}^{m} Q_{i} \neq \emptyset$. We attempt to find the feasible solution $z \in Q:=\bigcap_{i=1}^{m} Q_{i}$. We define a finite family of mappings $T_{i}: \mathbb{R}^{N} \rightarrow$ $\mathbb{R}^{N}(i=1,2, \cdots, m)$ by $T_{i} x:=P_{i}(x)\left(x \in \mathbb{R}^{N}\right)$, where $P_{i}(x)=P_{Q_{i}}(x)\left(x \in \mathbb{R}^{N}\right)$ stands for the metric projection from $\mathbb{R}^{N}$ onto $Q_{i}$. We define a mapping $S: \mathbb{R}^{N} \rightarrow \mathbb{R}^{N}$ by $S(x):=x^{2}\left(x \in \mathbb{R}^{N}\right)$. We observe that $T_{i}(i=1,2, \cdots, m)$ is quasi- $\phi_{p}$-nonexpansive with respect of $F i x\left(T_{i}\right) \neq \emptyset$. In this experiment, we set $Q_{i}(i=1,2, \cdots, m)$ as a closed ball with center $c_{i} \in \mathbb{R}^{N}$ and radius $r_{i}>0$. Thus $P_{i}(i=1,2, \cdots, m)$ can be computed with

$$
P_{i}(x):= \begin{cases}c_{i}+\frac{r_{i}}{\left\|c_{i}-x\right\|}\left(x-c_{i}\right), & \text { if }\left\|c_{i}-x\right\|>r_{i}, \\ x, & \text { if }\left\|c_{i}-x\right\| \leq r_{i} .\end{cases}
$$

We choose $r_{i}=1(i=1, \ldots, m), c_{1}=(1,0, \ldots, 0)^{T}$ and $c_{2}=(-1,0, \ldots, 0)^{T}$. Meanwhile, $c_{i}$ is randomly generated in the range of $(-1 / \sqrt{N}, 1 / \sqrt{N})^{N}(i=3, \ldots, m)$. Give the values of $c_{1}, c_{2}, r_{1}$ and $r_{2}$ as above. In this case, one sees that $\Lambda=\bigcap_{i=1}^{m} F i x\left(T_{i}\right) \cap S^{-1}(0)=\bigcap_{i=1}^{m} Q_{i} \cap S^{-1}(0)=\mathbf{0} \neq \emptyset$.

We use our Algorithm (3.21) to solve Example 4.2, our parameter settings are given the same as in Example 4.1. Our initial value is randomly generated by the MATLAB function $k \times$ rand (Case I: $k=1$; Case II: $k=10$; Case III: $k=50$; Case IV: $k=100$ ). Let us denote $E_{n}=\left\|x_{n}-z\right\|^{2}$ by the iteration error of Algorithm (3.21) with $z=\mathbf{0}$. Let us take $E_{n}<10^{-4}$ as the stopping criteria. In addition, we also test the numerical behavior of our algorithm (3.21) with different values of $N$ and $m$. Our numerical results are reported in Figure 5-Figure 8.

Remark 4.1. (i) Figure 1, Figure 3, Figure 5 and Figure 7 show the computational performance of our proposed Algorithms (3.1) and (3.21) with different initial values and 


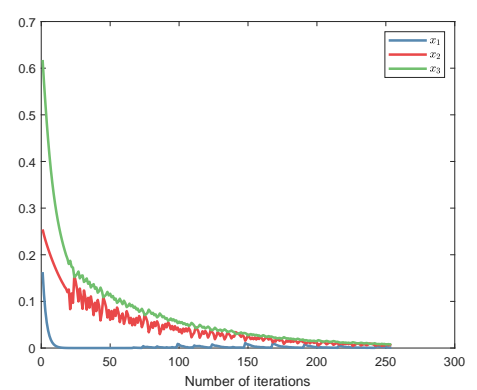

(A) Case I

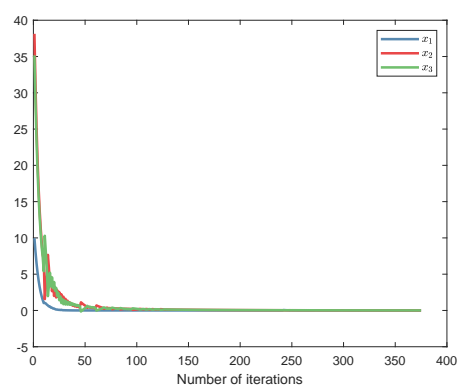

(C) Case III

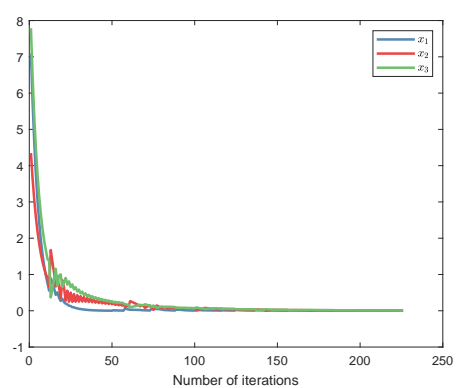

(B) Case II

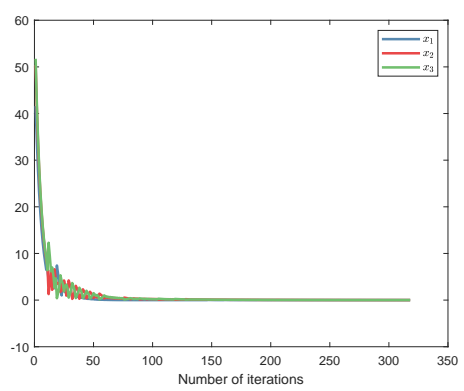

(D) Case IV

FIGURE 6. Numerical behaviors of each component of $x_{n}$ for Example 4.2.

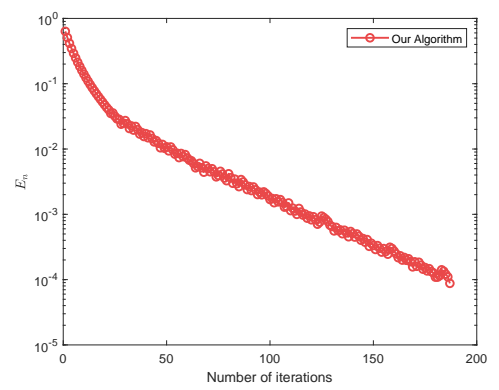

(A) $N=3, m=10$

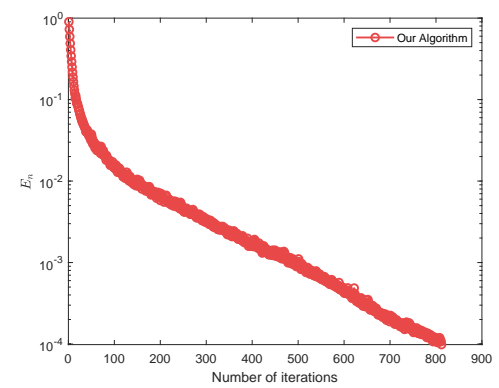

(C) $N=5, m=10$

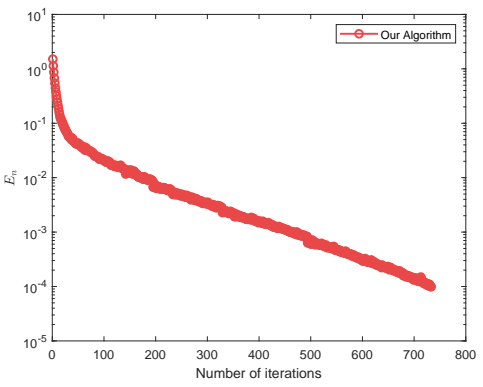

(B) $N=5, m=5$

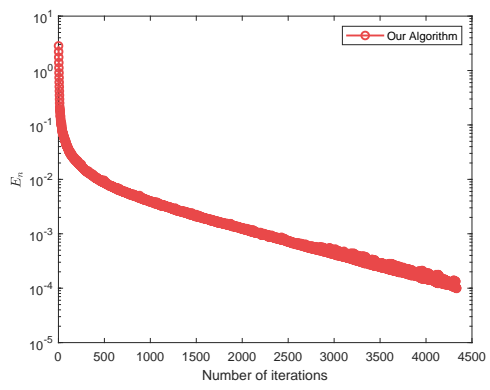

(D) $N=10, m=3$

FIGURE 7. Numerical behaviors of error sequence $\left\{E_{n}\right\}$ for Example 4.2. 


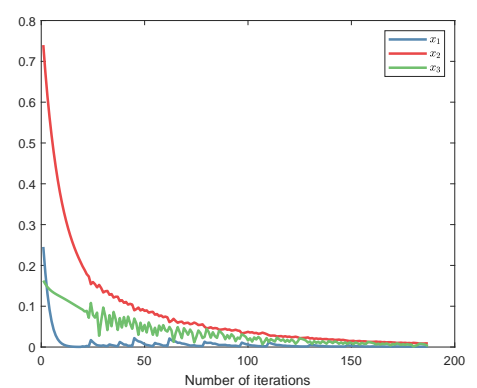

(A) $N=3, m=10$

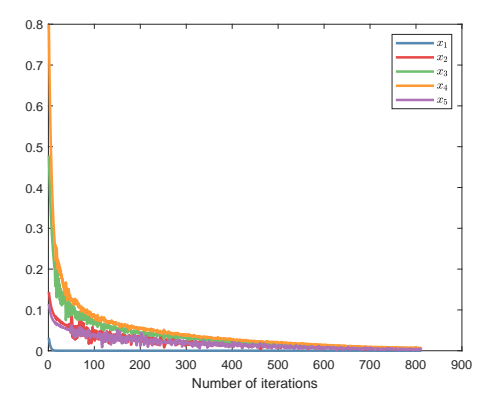

(C) $N=5, m=10$

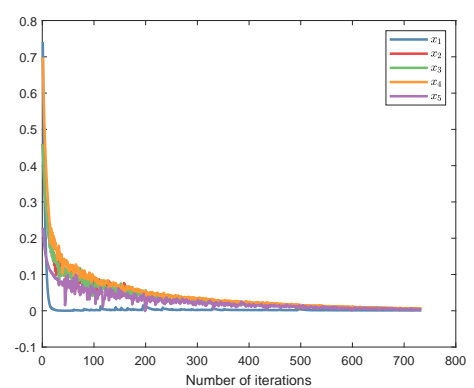

(B) $N=5, m=5$

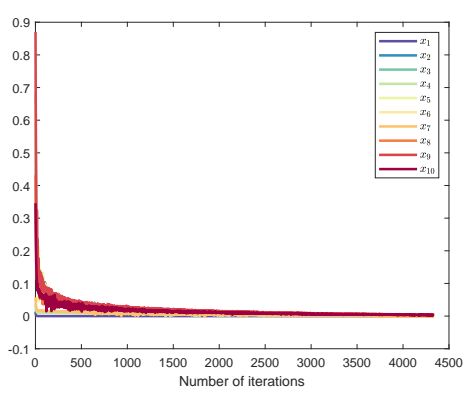

(D) $N=10, m=3$

FIGURE 8. Numerical behaviors of each component of $x_{n}$ for Example 4.2.

different dimensions. On the other hand, we also display the numerical behaviors of each component of the generated sequence $\left\{x_{n}\right\}$ with different initial values and different dimensions, see Figure 2, Figure 4, Figure 6 and Figure 8. The numerical results show that the generated sequence $\left\{x_{n}\right\}$ is convergent to 0 as $n \rightarrow \infty$. From which, it implies that the sequence $\left\{x_{n}\right\}$ converges to the solution of problems.

(ii) Based on the provided data in Example 4.1 and Example 4.2, we conclude that our proposed Algorithms (3.1) and (3.21) are efficient for solving optimization problems in finite dimensional Euclidean spaces. In addition, our algorithms are robust because the choice of initial values and dimensions do not affect the computational performance of the algorithms. 


\section{REFERENCES}

[1] Y.I. Alber, Metric and generalized projection operators in Banach spaces: properties and applications, Lect. Pure Appl. Math. pp. 15-50, 1996.

[2] L.M. Bregman, The relaxation method of finding the common point of convex sets and its application to the solution of problems in convex programming, USSR Comput. Math. Math. Phys. 7 (1967), 200-217.

[3] Y. Censor, A. Lent, An iterative row-action method for interval convex programming, J. Optim. Theory Appl. 34 (1981), 321-353.

[4] S.S. Chang, C.F. Wen, J.C. Yao, Zero point problem of accretive operators in Banach spaces, Bull. Malaysian Math. Sci. Soc. 42 (2019), 105-118.

[5] S.Y. Cho, A convergence theorem for generalized mixed equilibrium problems and multivalued asymptotically nonexpansive mappings, J. Nonlinear Convex Anal. 21 (2020), 1017-1026.

[6] S.Y. Cho, X. Qin, On hybrid projection methods for asymptotically quasi- $\phi$-nonexpansive mappings, Appl. Math. Comput. 215 (2010), 3874-3883.

[7] S.Y. Cho, X. Qin, L. Wang, Strong convergence of a splitting algorithm for treating monotone operators, Fixed Point Theory Appl. 2014 (2014), Article ID 94.

[8] I. Cioranescu, Geometry of Banach spaces, duality mappings and nonlinear problems, Springer Science \& Business Media, 2012.

[9] F. Kohsaka, W. Takahashi, Proximal point algorithms with Bregman functions in Banach spaces, J. Nonlinear Convex Anal. 6 (2005), 505-523.

[10] V. Martín Márquez, S. Reich, S. Sabach, Iterative methods for approximating fixed points of Bregman nonexpansive operators, Discrete Contin. Dyn. Syst. 6 (2013), 1043-1063.

[11] E. Naraghirad, J.C. Yao, Bregman weak relatively nonexpansive mappings in Banach spaces, Fixed Point Theory Appl., 2013 (2013), Article ID 141.

[12] P.P. Phelps, Convex functions, monotone operators and differentiability, Lecture Notes in Mathematics, Springer, Berlin, 2009.

[13] C.T. Pang, E. Naraghirad, Strong convergence of Halpern iterations for quasi-nonexpansive mappings and accretive operators in Banach spaces, Fixed Point Theory Appl, 2013 (2013), 332.

[14] X. Qin, S. Huang, T. Wang, On the convergence of hybrid projection algorithms for asymptotically quasi- $\phi$ nonexpansive mappings, Comput. Math. Appl. 61 (2011), 851-859.

[15] X. Qin, S.Y. Cho, L. Wang, Iterative algorithms with errors for zero points of m-accretive operators, Fixed Point Theory Appl. 2013 (2013), Article ID 148.

[16] X. Qin, S.Y. Cho, Convergence analysis of a monotone projection algorithm in reflexive Banach spaces, Acta Math. Sci. 37 (2017), 488-502.

[17] S. Reich, S Sabach, Two strong convergence theorems for Bregman strongly nonexpansive operators in reflexive Banach spaces, Nonlinear Anal. 73 (2010), 122-135.

[18] S. Reich, S. Sabach, A projection method for solving nonlinear problems in reflexive Banach spaces, J. Fixed Point Theory Appl. 9 (2011), 101-116.

[19] S. Reich, S. Sabach, Two strong convergence theorems for a proximal method in reflexive Banach spaces, Numer. Funct. Anal. Optim. 31 (2010), 22-44.

[20] S. Reich, S. Sabach, Existence and approximation of fixed points of Bregman firmly nonexpansive mappings in reflexive Banach spaces. In Fixed-Point Algorithms for Inverse Problems in Science and Engineering, Springer, pp. 301-316, New York, 2011.

[21] D.R. Sahu, J.C. Yao, M. Verma, K.K. Shukla, Convergence rate analysis of proximal gradient methods with applications to composite minimization problems, Optimization, (2020), 10.1080/02331934.2019.1702040.

[22] W. Takahashi, Y. Takeuchi, R. Kubota, Strong convergence theorems by hybrid methods for families of nonexpansive mappings in Hilbert spaces, J. Math. Anal. Appl. 341 (2008), 276-286.

[23] S. Takahahsi, W. Takahashi, The split common null point problem and the shrinking projection method in Banach spaces, Optimization, 65 (2016), 281-287.

[24] W. Takahashi, The split feasibility problem in Banach spaces, J. Nonlinear Convex Anal. 15 (2014), 1349-1335.

[25] B. Tan, S. Xu, S. Li, Inertial hybrid and shrinking projection algorithms for solving variational inequality problems, J. Nonlinear Convex Anal. 21 (2020), 2193-2206. 
[26] B. Tan, S. Xu, S. Li, Modified inertial hybrid and shrinking projection algorithms for solving fixed point problems, Mathematics 8 (2020), Article ID 236.

[27] Z.B. Xu, G.F. Roach, Characteristic inequalities of uniformly convex and uniformly smooth Banach spaces, J. Math. Anal. Appl. 157 (1991), 189-210.

[28] H.K. Xu, Inequalities in Banach spaces with applications, Nonlinear Anal. 16 (1991), 1127-1138.

[29] H. Zhou, X. Qin, Fixed Points of Nonlinear Operators. Iterative Methods, De Gruyter, Berlin, 2020. 